\title{
Anatomy involved in the jugular foramen approach for jugulotympanic paraganglioma resection
}

\author{
Michelle M. Inserra, M.D., Markus Pfister, M.D., ANd Robert K. JaCkler, M.D. \\ Department of Otolaryngology/Head and Neck Surgery, Stanford University School of Medicine, \\ Stanford, California
}

\begin{abstract}
The goal in paraganglioma resection is to allow adequate exposure to remove the lesion while preserving cranial nerve function. Knowledge of the anatomy of the jugular foramen is crucial to this endeavor. In this report the authors describe a jugular foramen approach for the resection of glomus jugulare tumors in cases in which rerouting of the facial nerve can be avoided. This approach provides adequate exposure of the jugular bulb for many jugulotympanic paragangliomas without increased risk of injury to the facial nerve. In addition, special circumstances surrounding intracranial and carotid artery involvement are briefly discussed.
\end{abstract}

\author{
KEY WORDS - glomus jugulare tumor - jugular foramen - transjugular approach • \\ paraganglioma
}

Paragangliomas are tumors derived from the neural crest that arise from the extraadrenal paraganglia of the autonomic nervous system. The most common paraganglioma of the head and neck is the carotid body tumor, followed by the jugulotympanic paraganglioma. These are benign tumors that grow slowly, but they can be locally aggressive. Because of their insidious growth pattern, they are often not diagnosed until they reach an advanced stage. High-resolution computerized tomography scanning and magnetic resonance imaging are essential in the diagnosis and characterization of these lesions. Whereas magnetic resonance imaging is superior in its ability to define the relationship of the lesion to the surrounding vessels, computerized tomography scanning can help define osseus erosion by the tumor.

The risk of neurovascular injury is an important consideration in the treatment of these tumors. Therefore, definitive management is carefully considered with regard to the size and extent of the lesion, as are patient-related issues such as age, physical condition, and preoperative nerve function. Knowledge of the anatomical landmarks and surgical techniques is crucial to a successful resection.

\section{ANATOMY OF THE JUGULAR FORAMEN}

The jugular foramen lies at the junction of the base of the temporal and occipital bones. It has two compartments: the posterolateral pars venosa, which contains the jugular bulb and the 10th and 11th cranial nerves; and the anteromedial pars nervosa, which contains the IPS and ninth cranial nerve. Occasionally a fibroosseous septum

Abbreviations used in this paper: $\mathrm{CA}=$ carotid artery; ICA = internal CA; IPS = inferior petrosal sinus. can be found between the jugular spine of the temporal bone and that of the occipital bone, separating the jugular foramen into these two compartments. More often, however, there is no septation and the jugular foramen exists as one compartment.

The lower cranial nerves always pass medially to the jugular bulb. Tumors arising in the jugular bulb therefore displace the lower cranial nerves medially by their growth, creating a more favorable position for nerve preservation during resection. ${ }^{3}$ The ninth cranial nerve enters the jugular foramen medially and is the most anterior of the cranial nerves. As it exits the skull base, it is positioned as the most anterior and lateral of the nerves. This lateral position makes it slightly more susceptible to injury during resection. The 11th cranial nerve exits the skull base as the most posterior and medial of these nerves. The 10th cranial nerve exits medially between the ICA and the internal jugular vein.

The IPS, which often consists of multiple channels, courses from the cavernous sinus and empties into the medial aspect of the jugular bulb. The position of the lower cranial nerves with respect to the IPS is variable. The IPS will often pass between the ninth nerve anteriorly and the 10th and 11th nerves posteriorly. ${ }^{7}$ Therefore, overpacking the IPS or cautery in this area can cause nerve injury.

\section{PATIENT PREPARATION}

Continuous monitoring of the seventh, ninth, 10th, 11 th, and 12 th cranial nerves should be used. ${ }^{2}$ As an alternative to an electrode placed in the vocalis muscle to monitor the 10th cranial nerve, an electromyography endotracheal tube (Medtronic Xomed, Inc., Jacksonville, FL) can 
also be used. For larger glomus jugulare tumors, embolization is recommended approximately 24 to 48 hours prior to surgery. Glomus jugulare tumors are typically supplied by the following arteries: the ascending pharyngeal, occipital, internal maxillary, and/or ICA. Preoperative embolization has been shown to reduce bleeding, thereby improving visualization of the tumor and decreasing surgical time. ${ }^{4}$

\section{SURGICAL TECHNIQUE}

A curvilinear incision is made approximately three fingerbreadths behind the ear and is gradually curved into a horizontal neck crease parallel to the jaw line. A limited neck dissection is performed. The sternocleidomastoid muscle is reflected posteriorly and the digastric and styloid muscles are reflected anteriorly from the mastoid tip. The neurovascular components of the neck are then exposed and identified. The internal jugular vein and ICA are loosely encircled with a vascular loop. Dissection then continues superiorly toward the skull base, identifying and ligating the ascending pharyngeal and occipital arteries that also supply the tumor. The greater auricular nerve has to be sacrificed, with resulting hypesthesia to the lower portion of the external ear. This nerve should be dissected inferiorly, exposing it for some length, and then it should be divided sharply and atraumatically; it can then be preserved for a possible nerve graft. ${ }^{1}$

Mastoidectomy is performed after identification of the sigmoid sinus posteriorly, the tegmen mastoideum superiorly, and wide exposure of the antrum anteriorly. The impression of the digastric muscle in the inferior portion of the mastoid (digastric ridge) is then identified and defined anteriorly. The facial nerve lies medial to the fascia of the digastric muscle at the level of the stylomastoid foramen and runs perpendicular to the ridge at its anterior edge. The fallopian canal is then skeletonized with a diamond burr to allow exposure of the jugular foramen. A thin, osseous shell can be left on the nerve. Once the nerve is defined, the retrofacial air cells are drilled to gain exposure of the sigmoid sinus. Proximally, a shell of bone is left over the sigmoid sinus to allow for extraluminal packing and occlusion of the sinus (Fig. 1). When the tumor has an intracranial component, however, the sinus is totally decompressed to achieve adequate exposure for ligation of the vessel. Ligation requires dural opening and passage of the ligature through the posterior fossa.

Moving inferiorly in the mastoid region, dissection now continues anterior to the facial nerve to expose the jugular bulb anteriorly. A diamond burr is used to drill the hypotympanic bone. To do this, the drill needs to approach the hypotympanum from both a lateral and a medial aspect with regard to the fallopian canal (Fig. 2). In this manner the fallopian canal is isolated all the way around, creating a bridge over the jugular bulb (Fig. 3). ${ }^{6}$

Proximal control of the sigmoid sinus is achieved with extraluminal packing consisting of Surgicel. Early occlusion of the sigmoid sinus is recommended to decrease blood flow to the tumor. Distal occlusion of the jugular vein in the neck, however, is best accomplished just before the tumor is ready to be removed, to avoid backpressure and increased bleeding.

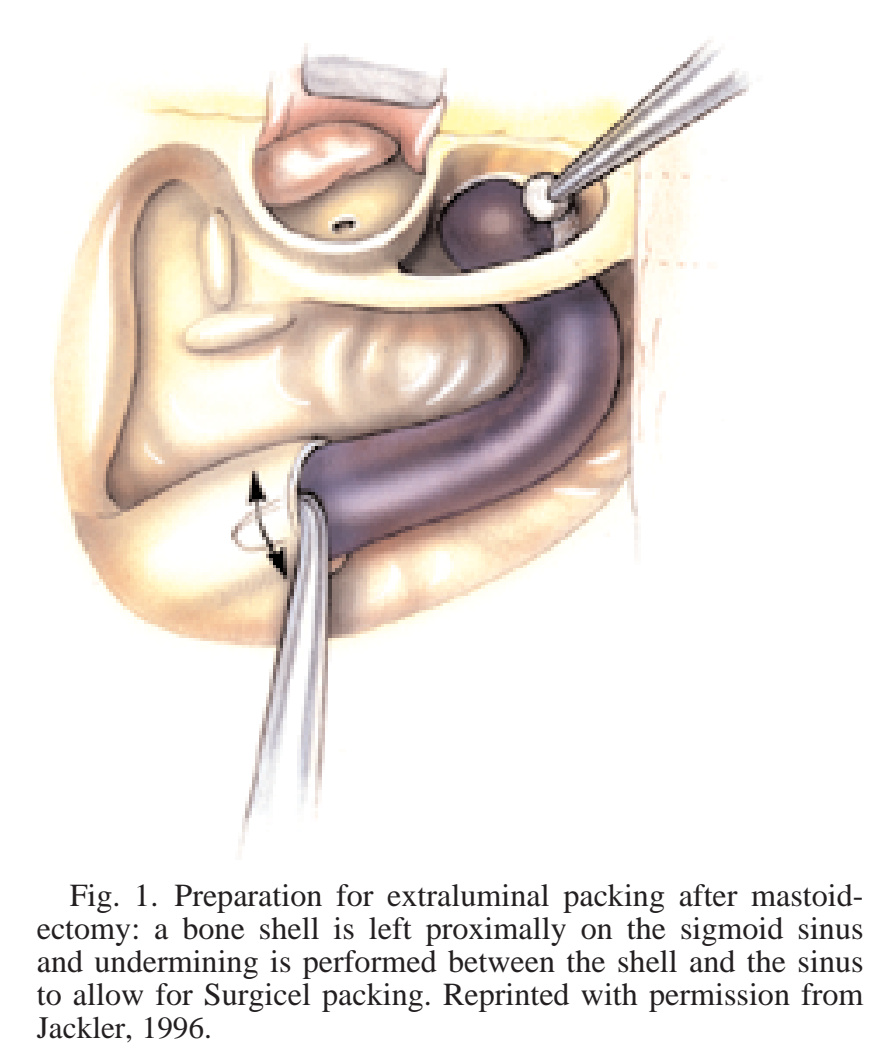

The sigmoid sinus is then opened sharply with a No. 11 blade (Fig. 3). The intraluminal tumor is dissected from the intima of the sinus and jugular bulb (Fig. 4). Characteristically, the tumor is fused to the wall of the jugular bulb, but lies free in the lumen of the sigmoid sinus and jugular vein. At this point, bleeding from the IPS and condylar vein is encountered. This is best controlled by firm placement of Surgicel over the orifices of the IPS. Care should be taken to avoid tight packing into the openings of this structure because this can potentially injure the ninth, 10th, and 11th cranial nerves, which lie just medial to the jugular bulb.

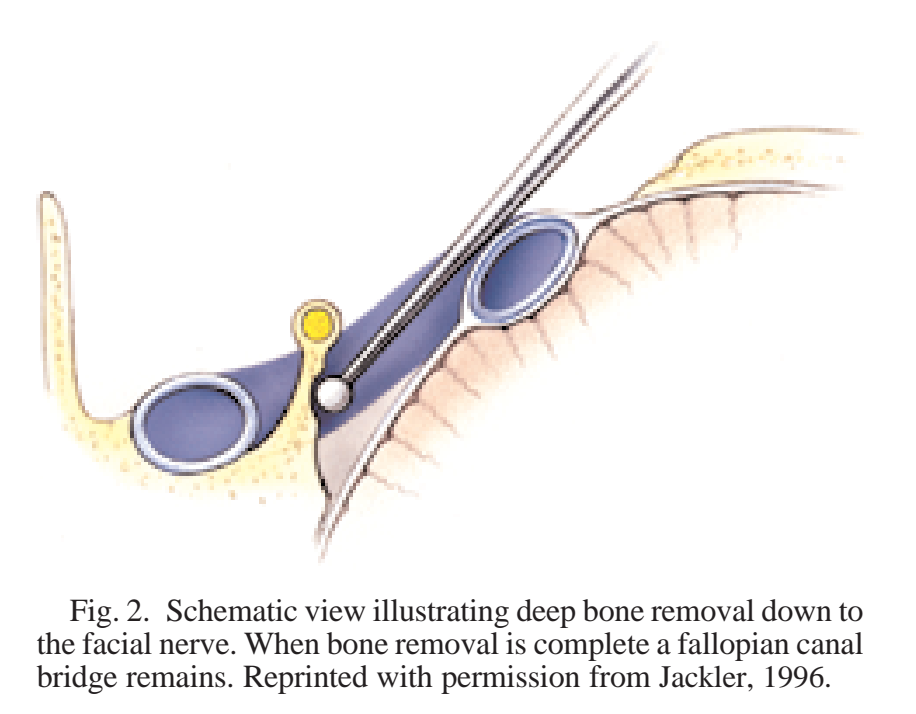




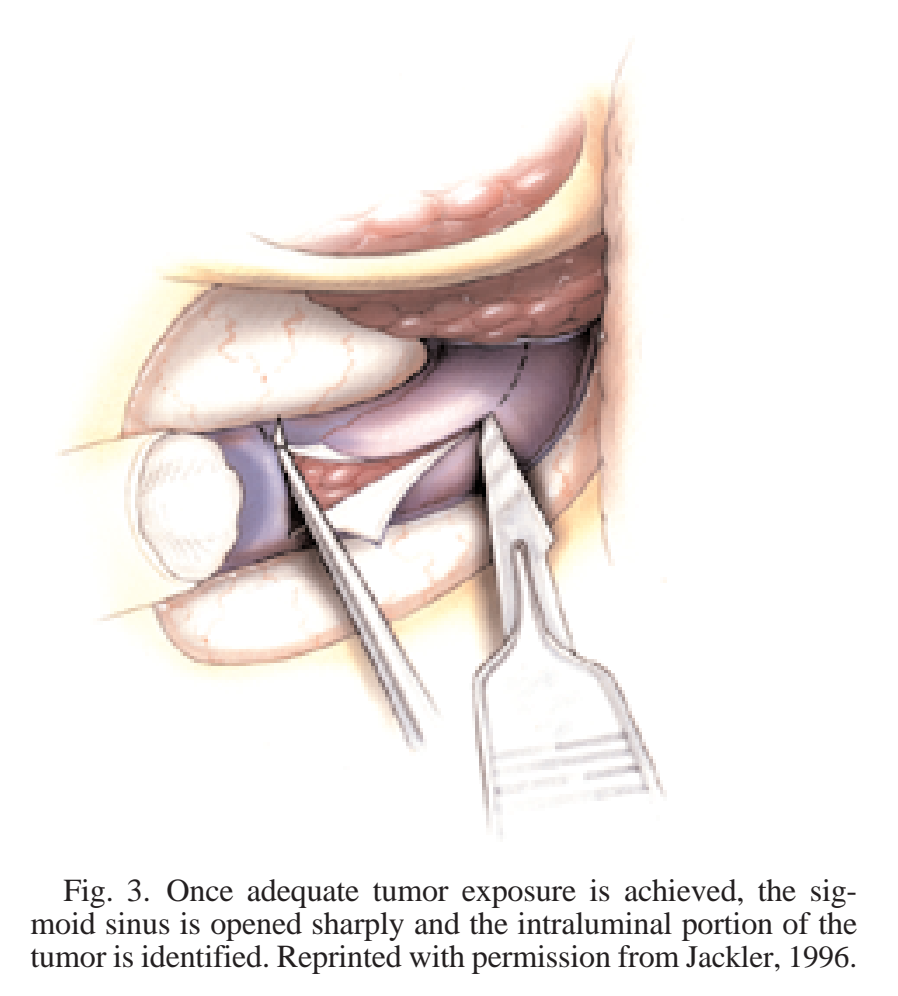

At this point, ear canal incisions are made and a tympanomeatal flap is raised inferiorly to expose the hypotympanum of the middle ear. Wide exposure of the hypotympanum is achieved. The middle ear component of the lesion can then be removed using tympanotomy or deliv-

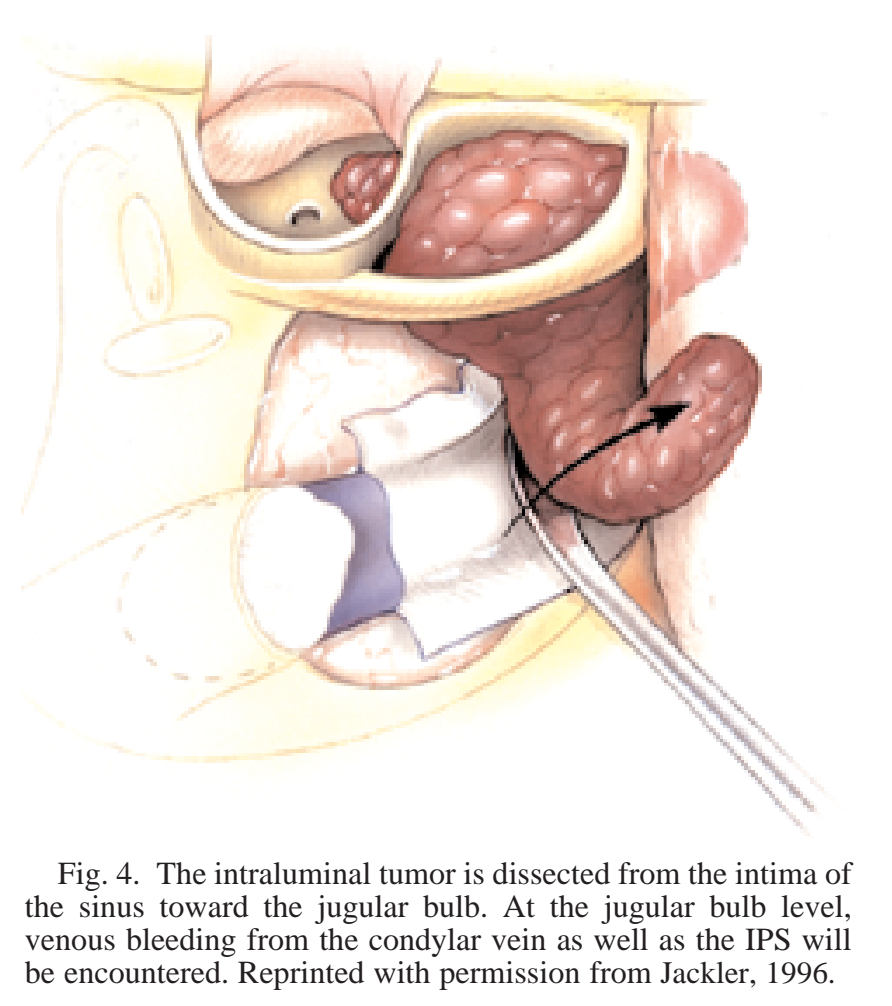

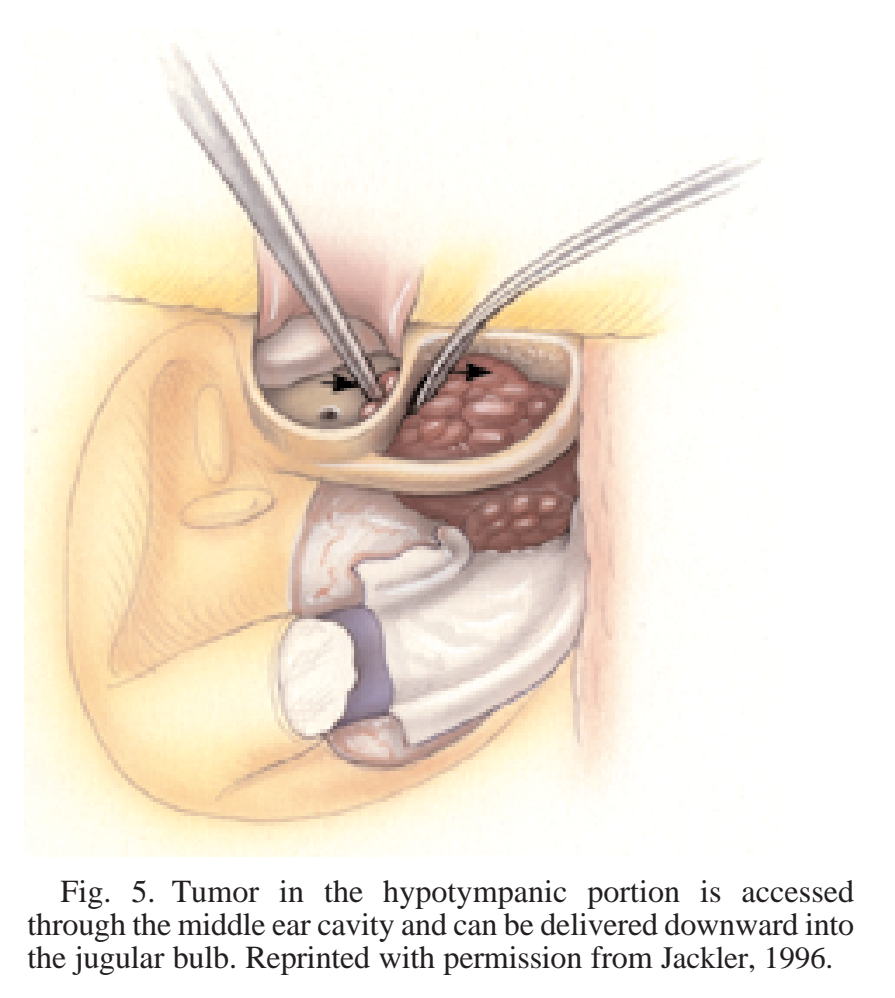

ered into the jugular fossa with the main bulk of the tumor (Fig. 5). When the lesion is entwined with the ossicles, use of a visible-spectrum laser (for example, Argon) facilitates atraumatic removal. The remainder of the tumor, which generally has grown beyond the confines of lumen in the region of the jugular bulb, can then be removed.

In cases in which a jugular foramen tumor has an intracranial component, a transjugular craniotomy is performed and the sigmoid sinus is ligated rather than extraluminally compressed. An additional 1 to $2 \mathrm{~cm}$ of retrosigmoid bone is removed to expose the posterior fossa dura. A ligature can then be passed between the medial surface of the sigmoid sinus and the lateral lobe of the cerebellum (Fig. 6). In our experience, most patients with jugular foramen tumors with intracranial extension can be treated with a single-stage transjugular craniotomy. Facial nerve mobilization or ear canal closure is usually not required, permitting conservation of facial function and hearing, when these are present preoperatively. ${ }^{5}$ It must be stressed that to avoid untoward hemorrhage, the intracranial component should be resected only after exenterating the cranial base component. Fortunately, the intracranial portions of paragangliomas characteristically possess little adherence to adjacent brain. The tumor is dissected from the filamentous branches of the lower cranial nerves with great care. Only when adequate hemostasis has been achieved through embolization, ligation, occlusion of venous channels with packing, and judicious bipolar cautery is orderly microdissection of the tumornerve interface possible.

In cases in which the tumor extensively erodes the CA wall, the facial nerve needs to be rerouted and a canal wall mastoidectomy performed to obtain adequate exposure. In such cases, the ear canal and middle ear are removed and 


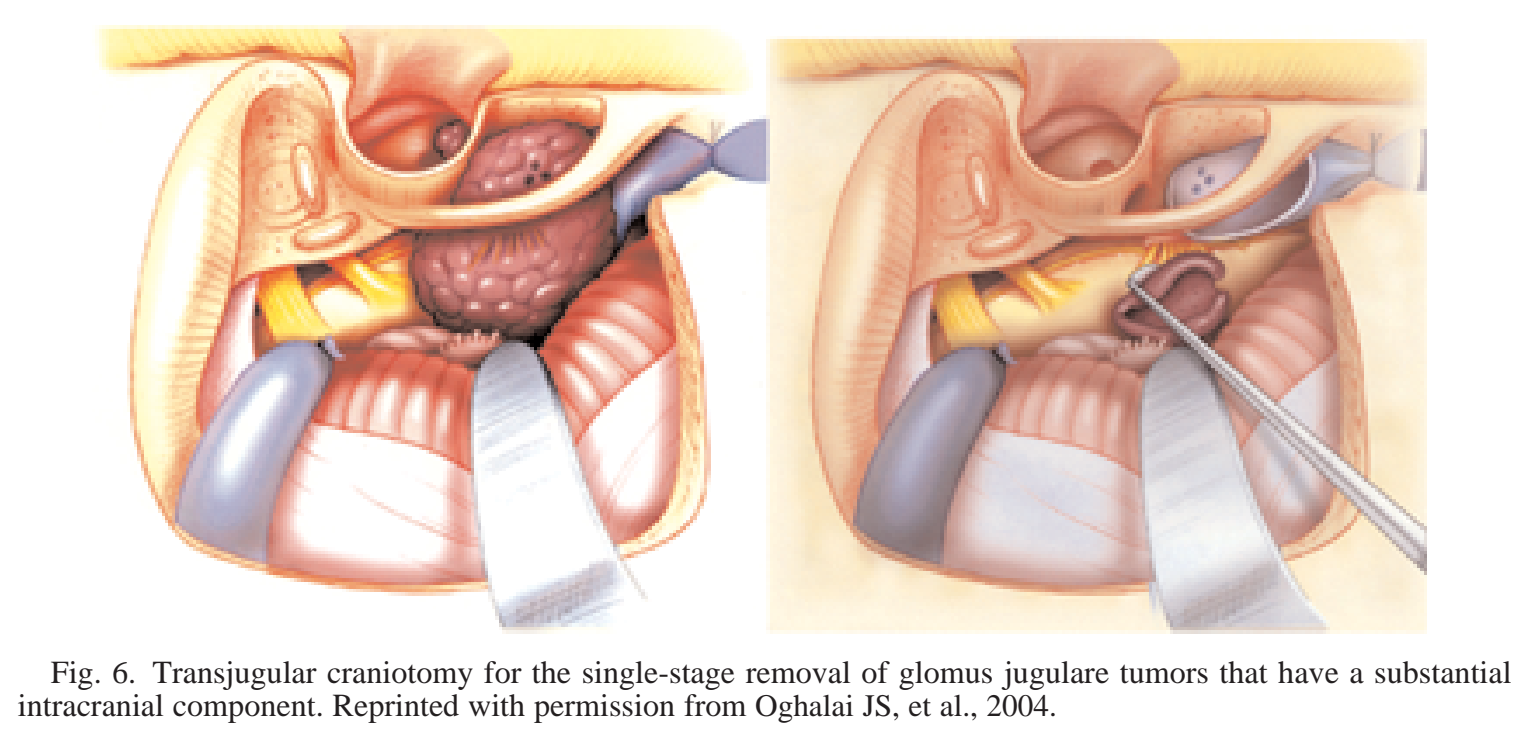

the meatus is sewn shut over an obliterating adipose tissue graft. Tumor is then carefully dissected from the CA. When it becomes unsafe to remove more tumor, the residual remnants should be well cauterized. In our experience, small remnants of paraganglioma left on the CA wall have a limited potential for generating a recurrence.

Fat is harvested from the left lower quadrant of the abdomen along with a small piece of rectus fascia. The fat is soaked in Kefzol solution, cut into strips, and then placed in the wound. The incisions are closed in three layers. A standard mastoid compression dressing is then applied.

\section{References}

1. Jackler RK: Atlas of Neurotology and Skull Base Surgery. St. Louis: Mosby, 1996

2. Lanser MJ, Jackler RK, Yingling CD: Regional monitoring of the lower (ninth through twelfth) cranial nerves, in Kartush J, Bouchard K (eds): Neuroimaging in Otology and Head and Neck Surgery. New York: Raven Press, 1992, pp 131-150
3. Lustig LR, Jackler RK: The variable relationship between the lower cranial nerves and jugular foramen tumors: implications for neural preservation. Am J Otol 17:658-668, 1996

4. Murphy TP, Brackmann DE: Effects of preoperative embolization on glomus jugulare tumors. Laryngoscope 99:1244-1247, 1989

5. Oghalai JS, Leung MK, Jackler RK, et al: Transjugular craniotomy for the management of jugular foramen tumors with intracranial extension. Otol Neurotol 25:570-579, 2004

6. Pensak ML, Jackler RK: Removal of jugular foramen tumors: the fallopian bridge technique. Otolaryngol Head Neck Surg 117:586-591, 1997

7. Sen C, Hague K, Kacchara R, et al: Jugular foramen: microscopic anatomic features and implications for neural preservation with reference to glomus tumors involving the temporal bone. Neurosurgery 48:838-848, 2001

Manuscript received May 17, 2004.

Accepted in final form July 14, 2004.

Address reprint requests to: Robert K. Jackler, M.D., Department of Otololaryngology, Head and Neck Surgery, Stanford University Medical Center, 300 Pasteur Drive, Stanford, California 94305. email: jackler@stanford.edu. 